\title{
Potenciales efectos proarrítmicos de la farmacoterapia contra SARS-CoV-2
}

\author{
Potential proarrhythmic effects of pharmacotherapy against SARS-CoV-2
}

\author{
Argelia Medeiros-Domingo,,$*$ Omar F Carrasco, ${ }^{\S}$ Ana Berni-Betancourt ${ }^{\ddagger}, \|$
}

\footnotetext{
Palabras clave: SARS-CoV-2, tratamiento COVID-19, síndrome de QT largo, cloroquina, azitromicina, pandemia.

Keywords: SARS-CoV-2, COVID-19 treatment, Long QT syndrome, chloroquine, azithromycin, pandemic.
}

\footnotetext{
* Director Médico. Swiss DNAlysis, Dübendorf/Suiza \$ Sociedad Mexicana de Cardiología. Ciudad de México, México.

$\S$ Departamento de Farmacología y Centro de Investigación en Políticas, Población y Salud, Facultad de Medicina de la UNAM. Ciudad de México, México.

\| Laboratorio de Arritmias. Departamento de Cardiología del Hospital Central Sur de Alta Especialidad (HCSAE) PEMEX. Ciudad de México, México.

Recibido: 20/04/2020

Aceptado:

29/04/2020
}

RESUMEN

La pandemia por el virus SARS-CoV-2 causante de la enfermedad COVID-19 representa un reto mundial dada su alta tasa de transmisión y ausencia de una terapia efectiva o vacuna. Este escenario ha propiciado el uso de diversos fármacos que in vitro han demostrado un posible efecto contra el virus. Sin embargo, el tiempo no ha sido suficiente para evaluar su efectividad clínica con el adecuado rigor científico que precede a la prescripción de medicamentos. El uso de cloroquina/hidroxicloroquina, azitromicina y esquemas antivirales ha sido propuesto por diversos grupos, apoyado por una serie de pacientes limitada en número. Si bien puede representar la única esperanza para muchos enfermos, es importante conocer los principales efectos adversos asociados al uso de estas drogas y seleccionar mejor a los pacientes que puedan beneficiarse de ellas. El riesgo de arritmias ventriculares incrementa tanto por el uso de fármacos como por la gravedad de la propia enfermedad viral.

\section{ABSTRACT}

The pandemic caused by the SARS-CoV-2 or COVID-19 virus has been a global challenge given its high rate of transmission and lack of effective therapy or vaccine. This scenario has led to the use of various drugs that have demonstrated a potential effect against the virus in vitro. However, time has not been enough to properly evaluate their clinical effectiveness. The use of chloroquine/ hydroxychloroquine, azithromycin and antiviral treatment and has been proposed by various groups, supported by in vitro studies and limited patient series, without the adequate scientific rigor that precedes drug prescription. Although it may represent the only hope for many patients, it is important to know the main adverse effects associated with the use of these drugs and to better select patients who may benefit from them.

\section{INTRODUCCIÓN}

$E^{n}$ n los últimos meses hemos presenciado la pandemia más grave de la era «moderna» asociada al coronavirus 2 (SARS-CoV-2), dada su alta contagiosidad, según cifras oficiales (John Hopkins University) ha cobrado la vida de cerca de 150,000 personas alrededor del mundo (abril, 2020). Este número de defunciones es un mínimo aproximado, pues los casos sospechosos sin prueba confirmatoria no han sido contabilizados. En medio de este gran reto y sin contar aún con una vacuna, se han utilizado múltiples fármacos en corto tiempo y sin el adecuado protocolo requerido para evaluar científicamente su verdadera utilidad en el tratamiento de los pacientes. La enfermedad por coronavirus COVID-19 se manifiesta por fiebre y síntomas respiratorios; la severidad de los síntomas y la mortalidad es mayor en pacientes con enfermedad cardiovascular prexistente. ${ }^{1}$ De acuerdo con los casos reportados de Wuhan, China, las complicaciones cardiacas en pacientes que requirieron atención en cuidados intensivos son choque cardiogénico (30.6\%), arritmias (44.4\%) y daño miocárdico agudo (7.2\%). ${ }^{2}$ Los esquemas de tratamiento actual que parecieran ser de utilidad en COVID-19 son cloroquina/hydroxicloroquina, azitromicina, antivirales (lopinavir/ritonavir) y agentes inmunológicos reguladores de inflamación. En espera de estudios más convincentes, en esta breve revisión se describen las propiedades farmacológicas de los medicamentos así como su posible efecto proarrítmico. Se hace un llamado al uso cauteloso de estas drogas que 
pueden afectar gravemente la repolarización ventricular y condicionar arritmias mortales en algunos pacientes. ${ }^{3}$

\section{PRINCIPALES FÁRMACOS UTILIZADOS EN EL TRATAMIENTO DE COVID-19}

\section{Cloroquina/hidroxicloroquina}

La cloroquina y su análogo estructural hidroxicloroquina son agentes antimaláricos con cualidades terapéuticas para enfermedades reumatológicas. ${ }^{4}$ Tiene también potentes efectos inmunomoduladores, entre los que destaca la reducción de citosinas como interleucina 1 y 6 (IL-1, IL-6), factor de necrosis tumoral (TNF) e interferón (IFN). ${ }^{2}$ Se ha demostrado in vitro actividad contra virus ARN (rabia, poliovirus, dengue, ébola) y coronavirus (SARS-CoV-1 y MERS-CoV) y actualmente utilizado para la enfermedad COVID-19. Su efecto antiviral ocurre al limitar la unión de las partículas virales a la superficie celular, prevención de endocitosis, supresión de la fusión del virus con endosoma y limitación del proceso de maduración del virus. ${ }^{5}$ Los eventos adversos asociados incluyen trastornos de la repolarización ventricular (QT prolongado) con mayor susceptibilidad a desarrollar arritmias ventriculares graves, del tipo de torsade de pointes o torcida de puntas, fibrilación ventricular y muerte súbita. Este efecto es dosis dependiente y se debe al bloqueo del canal de potasio $\mathrm{KCNH} 2$. Otros efectos adversos incluyen prurito, náusea, cefalea, hipoglicemia y alteraciones neuropsiquiátricas. La hidroxicloroquina es metabolizada en el hígado por el citocromo CYP3A4. ${ }^{6}$

\section{Azitromicina}

Es un macrólido utilizado para diversas enfermedades bacterianas. Se ha explorado su uso en modelos de infecciones virales como virus sincitial respiratorio, donde se observó la reducción de inflamación de las vías respiratorias y de los niveles de citosinas como interleucina 5 y 6 (IL-5, IL-6) e interferón-Gamma. ${ }^{7}$ Se ha demostrado actividad in vitro contra diversos virus, incluyendo el virus de influenza $(\mathrm{H} 1 \mathrm{~N} 1),{ }^{8}$ virus del $\mathrm{Zika}^{9}$ y recientemente
SARS-CoV-2. Se elimina sin cambios en heces a través de excreción biliar. Su biotransformación hepática es mínima y, a diferencia de otros macrólidos, no se metaboliza ni inhibe al citocromo P450 3A4. ${ }^{10}$ Los macrólidos son capaces de prolongar el intervalo QT e inducir arritmias ventriculares. ${ }^{11,12}$ Se ha propuesto que la exposición crónica a azitromicina incrementa la corriente de sodio $\left(\mathrm{Na}^{+}\right)$, lo que propicia la prolongación del intervalo QT y arritmias cardiacas. ${ }^{1}$

\section{Lopinavir/ritonavir}

Es la combinación de inhibidores de proteasa útil en el tratamiento de infección por $\mathrm{VIH}$. Se ha descrito actividad in vitro y en animales contra otros coronavirus como SARS y MERS. ${ }^{14}$ Las publicaciones iniciales de lopinavir/ritonavir como esquema terapéutico de COVID-19 no han mostrado diferencia en tiempo de mejoría clínica, disminución de carga viral o mortalidad a 28 días en pacientes con enfermedad severa. ${ }^{15}$ Su metabolismo es rápido y extenso en el hígado a través de la vía CYP3A4. Se recomienda usar con precaución debido a sus múltiples interacciones con otros fármacos y posibles efectos secundarios. Las reacciones adversas más frecuentes son de origen gastrointestinal, diarrea, náusea y vómito. Por su influencia en la vía metabólica del CYP3A4 no se debe coadministrar con medicamentos que utilizan el CYP3A4 para su biotransformación. ${ }^{16}$

\section{Remdesivir}

Es un antiviral de reciente creación que pertenece a la familia de análogos de nucleótidos y que inhibe la ARN polimerasa viral. Ha demostrado actividad in vitro en contra de SARSCoV-2 y otros coronavirus como SARS y MERS$\mathrm{CoV} .{ }^{16}$ Se ha sido descrito el uso de remdesivir para el tratamiento de COVID-19 en humanos. Un estudio reciente de uso compasivo en 53 pacientes con enfermedad COVID-19 grave demostró $68 \%$ de mejoría ventilatoria, $47 \%$ de los pacientes fueron dados de alta y $13 \%$ murieron. Las reacciones adversas observadas fueron aumento de enzimas hepáticas, diarrea, insuficiencia renal, hipotensión y disfunción orgánica múltiple. ${ }^{17}$ 
Inhibidores de interleucina 6 (IL-6)

En pacientes con enfermedad COVID-19 severa se han descrito escenarios clínicos consistentes con el síndrome de liberación de citosinas con elevación de IL-6; existen reportes anecdóticos con buenos desenlaces clínicos tras la administración de tocilizumab (anticuerpo monoclonal recombinante humanizado, antagonista del receptor de IL-6). ${ }^{18} \mathrm{Su}$ administración subcutánea e intravenosa ha demostrado utilidad en el tratamiento de artritis reumatoide. Las reacciones adversas observadas incluyen infecciones del tracto respiratorio alto, nasofaringitis y neumonía, perforación gastrointestinal, accidente vascular cerebral e infarto al miocardio. Se ha reportado también neutropenia, aumento de enzimas hepáticas e hiperlipidemia. ${ }^{19}$

\section{MECANISMOS ARRITMOGÉNICOS EN EL PACIENTE GRAVE COVID-19}

Las complicaciones cardiovasculares como daño miocárdico, choque y arritmias son frecuentes en el paciente COVID. ${ }^{20}$ Las arritmias ventriculares malignas como taquicardia/fibrilación ventricular (TV/FV) se presentan hasta en $5.9 \%$ de los pacientes en estado crítico. Los factores que predisponen a la presencia de arritmias se describen a continuación:

\section{Daño miocárdico}

El daño directo al tejido cardiaco se manifiesta por elevación de troponinas y puede acompañarse de síntomas como palpitaciones y dolor precordial. El nivel de troponinas y otros biomarcadores es significativamente más alto en pacientes en estado crítico y es factor pronóstico de mortalidad. ${ }^{21}$ La incidencia de TV/FV es mayor en pacientes con elevación de troponinas; además del daño miocárdico pueden coexistir otros mecanismos arritmogénicos como hipoxia, inflamación y citotoxicidad directa del virus.

\section{Tormenta de citoquinas inflamatorias}

Consiste en una respuesta inflamatoria severa derivada de la liberación exagerada y fulminante de citoquinas que culmina en la falla orgánica múltiple. ${ }^{22} \mathrm{El}$ perfil bioquímico predictor de mortalidad es la elevación de ferritina y niveles séricos de interleucina. (IL6). ${ }^{23}$ En este escenario es frecuente observar alargamiento del intervalo QT y arritmias ventriculares como torsade de pointes (TdP); el sustrato electrofisiológico es un aumento en la duración del potencial de acción secundario a trastornos en la expresión o funcionamiento de canales iónicos de $\mathrm{K}^{+}$y $\mathrm{Ca}^{++}$por liberación excesiva de IL-6, IL-1 y factor de necrosis tumoral $(\mathrm{TNF} \alpha) .{ }^{24}$ Existe también evidencia que asocia la liberación de citoquinas proinflamatorias con hiperactividad del sistema simpático, con un efecto mediado por vía central (hipotálamo) y periférica (activación de ganglio estrellado), lo que incrementa el riesgo de arritmias ventriculares. ${ }^{25}$

\section{Arritmias en paciente en estado crítico}

El enfermo en estado crítico tiene mayor susceptibilidad a presentar arritmias por las condiciones propias del evento agudo: alteraciones hidroelectrolíticas, sepsis, isquemia miocárdica, falla cardiaca, bradicardia, enfermedad renal, uso concomitante de fármacos como sedantes, analgésicos, antieméticos, antibióticos y aminas vasoactivas. Es importante considerar la concentración plasmática y ajuste de dosis de fármacos concomitantes en el paciente $\mathrm{CO}$ VID, ya que la incidencia de función hepática anormal puede ser hasta $51 \%$ en pacientes que reciben esquemas antivirales. ${ }^{26}$ La combinación de dos o más fármacos con efecto proarrítmico conocido incrementa el riesgo de prolongar el QT; sin embargo, el riesgo de TdP inducido por fármacos es variable y depende de cada medicamento en particular.

\section{FACTORES ASOCIADOS A SUSCEPTIBILIDAD INDIVIDUAL A DESARROLLAR ARRITMIAS CARDIACAS CON EL USO DE MEDICAMENTOS}

\section{Síndrome de QT largo}

El síndrome de QT largo (SQTL) se caracteriza por una grave alteración congénita en la repolarización ventricular traducida en pro- 
longación del intervalo QT y susceptibilidad a desarrollar arritmias ventriculares graves y muerte súbita. ${ }^{27}$ Hoy en día, se reconocen tres principales genes asociados a la enfermedad: KCNQ1 que codifica el canal que genera la corriente de repolarización lenta Iks; $\mathrm{KCNH} 2$ que codifica el canal que genera la corriente rápida de potasio IKr; y SCN5A que codifica canal de sodio encargado de la corriente INa. Desde la descripción de la enfermedad se ha reconocido que estos pacientes son particularmente sensibles a medicamentos que afectan la repolarización ventricular y por ende, prolongan el intervalo QT. Para mayor información sobre medicamentos de riesgo se puede visitar el sitio: https://crediblemeds.org/pdftemp/pdf/ DrugsToAvoidList.pdf

Los tratamientos utilizados para COVID-19 como cloroquina/hidroxicloroquina y azitromicina son parte de esta lista, su administración en el contexto de SQTL puede condicionar graves arritmias ventriculares y muerte súbita, particularmente si se administran juntos, como algunos han propuesto, por lo que su administración en estos casos en particular debe ser en extremo cautelosa, limitarse a casos intrahospitalarios en los que se pueden monitorizar trastornos de ritmo y considerar cuidadosamente el riesgo/beneficio.

\section{QT prolongado inducido por fármacos}

La prolongación del intervalo QT inducida por fármacos (diSQTL) es una entidad particular en la que los pacientes son susceptibles a prolongar el intervalo QT con ciertos medicamentos, lo que da lugar a arritmias ventriculares graves. ${ }^{28,29}$ Estos pacientes suelen tener un intervalo QTC normal y desarrollan QTc largo sólo cuando son expuestos a medicamentos que afectan la repolarización ventricular. Un tercio de los casos puede tener una mutación no reconocida previamente. ${ }^{30}$ La susceptibilidad de presentar arritmias se debe a la reserva de repolarización ventricular reducida, misma que puede ser condicionada por diversos polimorfismos en canales iónicos que por sí mismos no generan SQTL congénito. El principal mecanismo de prolongación del intervalo QT es el bloqueo de la corriente de potasio Ikr, aunque se han descrito otros mecanismos. ${ }^{31}$

\section{SATURACIÓN DE LAS VÍAS DE ELIMINACIÓN DE FÁRMACOS}

Es importante considerar que diversos fármacos son metabolizados por las mismas enzimas y su asociación puede dar signos de intoxicación aun a dosis terapéuticas. La cloroquina y la hidroxicloroquina son metabolizadas principalmente por el citocromo P450 3A4 codificado por el gen CYP3A4 que es responsable del metabolismo de cerca de $50 \%$ de los medicamentos. Otros potentes inhibidores de esta enzima son: diltiazem, itraconazol, ketoconazol, ritonavir, verapamilo, warfarina, amiodarona, lidocaína y antirretrovirales. ${ }^{32}$ Diversos polimorfismos en el gen CYP3A4 reducen la actividad de la enzima, lo que confiere una susceptibilidad individual a los fármacos que son metabolizados por esta vía. ${ }^{33}$ Los casos portadores de estos polimorfismos pueden presentar signos de intoxicación incluso utilizando dosis convencionales. Dado que normalmente estos polimorfismos no son diagnosticados con la frecuencia deseada, es importante considerar ajustar la dosis de medicamentos en caso de tener pacientes que reciben múltiples drogas al mismo tiempo, común en el manejo de terapia intensiva, y estar atentos a los signos de intoxicación.

\section{RECOMENDACIONES FINALES DEL USO DE MEDICAMENTOS EN CASOS COVID-19}

1. Emplear la menor dosis efectiva, lo ideal es utilizar sólo un medicamento a la vez, y considerar que la asociación de hidroxicloroquina y azitromicina puede representar un riesgo, pues ambos fármacos prolongan el intervalo QT y los estudios en cuanto a la utilidad de esta asociación son muy limitados. Evitar la polifarmacia que pudiera saturar la vía de eliminación de estos medicamentos.

2. Lo ideal es realizar una medición basal del intervalo QT y corregirlo mediante la fórmula de Bazett antes de administrar cloroquina/hidroxicloroquina o azitromicina.

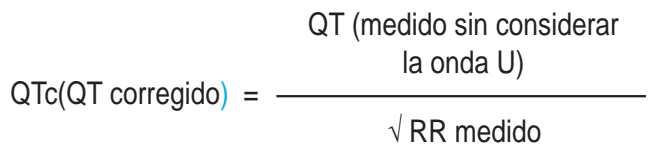


Los valores deben calcularse en segundos

3. El intervalo QTc debe medirse también durante el tratamiento. Se considera que un intervalo QTc mayor de 500 ms tiene alto riesgo de desarrollar arritmias ventriculares, el fármaco debe suspenderse o reducirse si se obtiene en cualquier momento esta cifra.

4. Los niveles séricos de potasio y magnesio deben mantenerse en niveles normales para evitar arritmias.

5. Otros factores como la hipoxemia y acidosis metabólica deben ser corregidos con prontitud, pues contribuyen al desarrollo de arritmias ventriculares en el contexto de prolongación del intervalo QT.

6. Evitar proporcionar múltiples medicamentos que se sabe prolongan el intervalo QT (lista en https://crediblemeds.org/pdftemp/ pdf/DrugsToAvoidList.pdf).

7. En pacientes con diagnóstico previo de SQTL, el uso de cloroquina/hidroxicloroquina $y / o$ azitromicina debe evaluarse cuidadosamente y con el tiempo, evitarse. Su administración, en caso necesario, debe llevarse a cabo en el contexto hospitalario.

\section{REFERENCIAS}

1. Huang C, Wang Y, Li X et al. Clinical features of patients infected with 2019 novel coronavirus in Wuhan, China. Lancet. 2020; 395: 497-506.

2. Wang D, Hu B, Hu C. Clinical characteristics of 138 hospitalized patients with 2019 novel coronavirusinfected pneumonia in Wuhan, China. JAMA. 2020; 323 (11): 1061-1069. doi: 10.1001/jama.2020.1585.

3. Juurlink DN. Safety considerations with chloroquine, hydroxychloroquine and azithromycin in the management of SARS-CoV-2 infection. CMAJ. 2020; 192 (17): E450-E453.

4. Shippey EA, Wagler VD, Collamer AN. Hydroxychloroquine: An old drug with new relevance. Cleve Clin J Med. 2018; 85 (6): 459-467. doi: 10.3949/ ccjm.85a.17034.

5. Devaux CA, Rolain JM, Colson P, Raoult D. New insights on the antiviral effects of chloroquine against coronavirus: what to expect for COVID-19? Int J Antimicrob Agents. 2020; 55 (5): 105938. doi: 10.1016/j. ijantimicag.2020.105938.

6. White NJ. Cardiotoxicity of antimalarial drugs. Lancet Infect Dis. 2007; 7 (8): 549-558. doi: 10.1016/S14733099(07)70187-1.

7. Mosquera RA, De Jesus-Rojas W, Stark JM et al. Role of prophylactic azithromycin to reduce airway inflammation and mortality in a RSV mouse infection model. Pediatr Pulmonol. 2018; 53 (5): 567-574. doi: 10.1002/ppul.23956.
8. Tran DH, Sugamata R, Hirose T, Suzuki S, Noguchi Y, Sugawara A et al. Azithromycin, a 15-membered macrolide antibiotic, inhibits influenza $A(H 1 N 1) p d m 09$ virus infection by interfering with virus internalization process. J Antibiot (Tokyo). 2019; 72 (10): 759-768.

9. Li C, Zu S, Deng YQ, Li D, Parvatiyar K, Quanquin N et al. Azithromycin protects against Zika virus infection by upregulating virus-induced type I and III interferon responses [published online ahead of print, 2019 Sep 16]. Antimicrob Agents Chemother. 2019; 63 (12): e00394-19.

10. Parnham MJ, Haber VE, Giamarellos-Bourboulis EJ, Perletti G, Verleden GM, Vos R. Azithromycin: Mechanisms of action and their relevance for clinical applications. Pharmacol Ther. 2014; 143 (2): 225-245. doi: 10.1016/j.pharmthera.2014.03.003.

11. Patel H, Calip GS, DiDomenico RJ, Schumock GT, Suda KJ, Lee TA. Prevalence of cardiac risk factors in patients prescribed azithromycin before and after the 2012 FDA Warning on the risk of potentially fatal heart rhythms. Pharmacotherapy. 2020; 40 (2): 107-115.

12. Albert RK, Schuller JL, Network CCR. Macrolide antibiotics and the risk of cardiac arrhythmias. Am J Respir Crit Care Med. 2014; 189 (10): 1173-180.

13. Yang Z, Prinsen JK, Bersell KR, Shen W, Yermalitskaya L, Sidorova T et al. Azithromycin causes a novel proarrhythmic syndrome. Circ Arrhythm Electrophysiol. 2017; 10 (4): e003560.

14. Chan JF, Yao Y, Yeung ML et al. Treatment with lopinavir/ritonavir or interferon- $\beta 1 \mathrm{~b}$ improves outcome of MERS-CoV infection in a nonhuman primate model of common marmoset. J Infect Dis. 2015; 212 (12): 1904-1913.

15. Cao B, Wang Y, Wen D et al. A trial of lopinavir-ritonavir in adults hos- pitalized with severe Covid-19. N Engl J Med. 2020. doi: 10.1056/nejmoa2001282.

16. Sanders J, Monogue M, Jodlowski T et al. Pharmacologic treatments for coronavirus disease 2019 (COVID-19) a review. JAMA. 2020; 323 (18): 1824-1836. doi: 10.1001/jama.2020.6019.

17. Grein J, Ohmagari N, Shin D et al. Compassionate use of remdesivir for patients with severe Covid-19. N Engl J Med. 2020. doi: 10.1056/NEJMoa2007016.

18. Mehta P, McAuley DF, Brown M et al. COVID-19: consider cytokine storm syndromes and immunosuppression. Lancet. 2020; 395 (10229): 1033-1034. doi: 10.1016/S0140-6736(20)30628-0.

19. Biggioggero M, Crotti C, Becciolini A, Favalli EG. Tocilizumab in the treatment of rheumatoid arthritis: An evidence-based review and patient selection. Drug Des Devel Ther. 2019; 13: 57-70. doi: 10.2147/DDDT. S150580.

20. Wang D, Hu B, Hu C. Clinical characteristics of 138 hospitalized patients with 2019 novel coronavirusinfected pneumonia in Wuhan, China. JAMA. 2020; 323 (11): 1061-1069. doi: 10.1001/jama.2020.1585.

21. Yang $X, Y u Y, X u J$ et al. Clinical course and outcomes of critically ill patients with SARS-CoV-2 pneumonia in Wuhan, China: a single-centered, retrospective, observational study [pub-lished correction appears in Lancet Respir Med. 2020; 8 (4): e26]. Lancet Respir Med. 2020; 8 (5): 475-481. doi: 10.1016/S22132600(20)30079-5. 
22. Ramos-Casals M, Brito-Zeron P, Lopez-Guillermo A et al. Adult haemophagocytic syndrome. Lancet. 2014; 383: 1503-1516.

23. Ruan Q, Yang K, Wang W et al. Clinical predictors of mortality due to COVID-19 based on an analysis of data of 150 patients from Wuhan, China. Intensive Care Med. 2020. doi: 10.1007/s00134-020-05991.

24. Lazzerini PE, Laghi-Pasini F, Boutjdir M, Capecchi PL. Cardioimmunology of arrhythmias: the role of autoimmune and inflammatory cardiac channelopathies. Nat Rev Immunol. 2019; 19 (1): 63-64.

25. Lazzerini PE, Capecchi PL, Laghi-Pasini F. Systemic inflammation and arrhythmic risk: lessons from rheumatoid arthritis. Eur Heart J. 2017; 38: 1717-1727.

26. National Health Commission and National Administration of Traditional Chinese Medicine of the People's Republic of China. Protocols for diagnosis and treatment of COVID-19. (7th Trial Version). (EB/OL)(202003-04) (2020-03-15).

27. Medeiros-Domingo A, Iturralde-Torres $P$, Ackerman MJ. Clinical and genetic characteristics of long QT syndrome. Rev Esp Cardiol. 2007; 60 (7): 739-752.

28. Sarganas G, Garbe E, Klimpel A, Hering RC, Bronder E, Haverkamp W. Epidemiology of symptomatic druginduced long QT syndrome and torsade de pointes in Germany. Europace. 2014; 16 (1): 101-108.

29. Arunachalam K, Lakshmanan S, Maan A, Kumar N, Dominic P. Impact of drug induced long qt syn-drome: a systematic review. J Clin Med Res. 2018; 10 (5): 384-390.

30. Itoh H, Crotti L, Aiba T, Spazzolini C, Denjoy I, Fressart $\mathrm{V}$ et al. The genetics underlying acquired long QT syndrome: impact for genetic screening. Eur Heart J. 2016; 37 (18): 1456-1464.

31. Kannankeril P, Roden DM, Darbar D. Drug-induced long QT syndrome. Pharmacol Rev. 2010; 62 (4): 760-781.

32. Berno G, Zaccarelli M, Gori C, Tempestilli M, Antinori A, Perno CF et al. Analysis of single-nucleotide polymorphisms (SNPs) in human CYP3A4 and CYP3A5 genes: potential implications for the metabolism of HIV drugs. BMC Med Genet. 2014; 15: 76.

33. Werk AN, Cascorbi I. Functional gene variants of CYP3A4. Clin Pharmacol Ther. 2014; 96 (3): 340-348.

Correspondencia:

Ana Berni-Betancourt

E-mail: dra.anaberni@gmail.com

\section{RESPONSABILIDADES ÉTICAS}

Protección de personas y animales. Los autores declaran que para esta investigación no se han realizado experimentos en seres humanos ni en animales.

Confidencialidad de los datos. Los autores declaran que han seguido los protocolos de su centro de trabajo sobre la publicación de datos de pacientes.

Derecho a la privacidad y consentimiento informado. Los autores declaran que en este artículo no aparecen datos de pacientes. 